\title{
Les Frères des Écoles chrétiennes et l’éducation de la foi
}

\section{Nive Voisine}

Volume 56, 1989

Changements culturels et éducation de la foi

Cultural Change and Education of the Faith

URI : https://id.erudit.org/iderudit/1006955ar

DOI : https://doi.org/10.7202/1006955ar

Aller au sommaire du numéro

Éditeur(s)

Les Éditions Historia Ecclesiæ Catholicæ Canadensis Inc.

ISSN

0318-6172 (imprimé)

1927-7067 (numérique)

Découvrir la revue

Citer cet article

Voisine, N. (1989). Les Frères des Écoles chrétiennes et l'éducation de la foi. Sessions d'étude - Société canadienne d'histoire de l'Église catholique, 56, 63-83. https://doi.org/10.7202/1006955ar

Tous droits réservés (C) Les Éditions Historia Ecclesiæ Catholicæ Canadensis Inc., 1989
Ce document est protégé par la loi sur le droit d'auteur. L'utilisation des services d'Érudit (y compris la reproduction) est assujettie à sa politique d'utilisation que vous pouvez consulter en ligne.

https://apropos.erudit.org/fr/usagers/politique-dutilisation/ 


\title{
Les Frères des Écoles chrétiennes et l'éducation de la foi
}

\author{
Nive VoISINE \\ Groupe de recherche sur les catéchismes \\ Université Laval, Québec
}

Fondé en 1680 par Jean-Baptiste de La Salle, l'Institut des Frères des Écoles chrétiennes élargit petit à petit son champ d'action - de Reims à Paris, puis dans divers diocèses de France, dans les pays limitrophes et enfin sur les cinq continents -, servi à la fois par un membership presque toujours à la hausse et par des circonstances moins heureuses comme les problèmes scolaires en France à la fin du XIX ${ }^{\mathrm{e}}$ siècle.

\section{Institut des Frères des Écoles chrétiennes Statistiques générales, 1710-1946}

$\begin{array}{lrrr} & \text { Maisons } & \text { Frères } & \text { Élèves } \\ 1710 & 17 & 190 & 7500 \\ 1760 & 105 & 715 & 24000 \\ 1810^{*} & 41 & 160 & 8800 \\ 1860 & 901 & 7787 & 309000 \\ 1910^{* *} & 714 & 10957 & 176679 \\ 1920 & 1199 & 11108 & 266174 \\ 1930 & 1269 & 13397 & 298713 \\ 1939^{* * *} & 1265 & 14415 & 323261 \\ 1946 & 1329 & 14207 & 390722\end{array}$

* L'institut a presque disparu pendant la Révolution française

** Effets des lois laïcistes en France

*** Dernière année avec des statistiques sûres

SOURCE: Rapports annuels et tableaux, Archives des Frères des Écoles chrétiennes, Maison généralice, Rome 
Malgré cette expansion et le déménagement de la maison mère à Lembecq-lez-Hal (Belgique) en 1904 et à Rome en 1937, la congrégation demeure, jusqu'à la Seconde guerre mondiale, essentiellement française et en grande partie gouvernée par des supérieurs français.

Arrivés à Montréal en novembre 1837, les Lasalliens se répandent rapidement partout en Amérique, aussi bien dans l'ensemble des ÉtatsUnis qu'au Canada (Ontario, Québec et, pour un temps, les Maritimes).

\section{Les Frères des Écoles chrétiennes au Canada}

\section{Statistiques, 1840-1946}

$\begin{array}{lrcr} & \text { Maisons } & \text { Frères } & \text { Élèves } \\ 1840 & 1 & 7 & 450 \\ 1860^{*} & 13 & 116 & 6970 \\ 1910 & 52 & 734 * * & 20388 \\ 1920 & 64 & 836 & \\ 1930 & 78 & 1090 & 28890 \\ 1939 & 93 & 1332 & 32579 \\ 1946 & 99 & 1346 & 32054 \\ \text { * Pour le Canada seulement } & \\ \text { ** Augmentation attribuable en grande partie à l'arrivée de frères venus de France } \\ \text { SOURCE: la même }\end{array}$

Un recrutement en progression presque continu - mais toujours insuffisant aux yeux des autorités lasalliennes - et l'appui d'un milieu favorable expliquent cette expansion. Très tôt, particulièrement au $\mathrm{XX}^{\mathrm{e}}$ siècle, les frères débordent l'enseignement primaire, leur premier champ d'apostolat, pour développer le secteur secondaire - spécialement dans leurs «grands pensionnats»: le Mont-Saint-Louis de Montréal, l'Académie commerciale de Québec, l'Académie De-La-Salle d'Ottawa, le De La Salle Institute de Toronto, l'Académie De-La-Salle de TroisRivières... - et font sentir leur présence même au niveau universitaire: l'Institut botanique de Montréal avec le frère Marie-Victorin, l'Institut pédagogique Saint-Georges de Montréal, l'École de Commerce de Québec, le St. Joseph's College d'Edmonton...

Des multiples activités des Frères des Écoles chrétiennes au Canada, je ne veux retenir qu'un seul aspect: leur rôle dans l'éducation de la foi, qui est au cœur de leur vocation de religieux. Je l'étudie en privilégiant l'approche du Groupe de recherche sur la production des catéchismes, 
c'est-à-dire en mettant l'accent sur leur fonction de catéchistes, d'intermédiaires entre les «producteurs» officiels et les jeunes «auditeurs». ${ }^{1}$ Dans une première partie, je vais exposer comment l'Institut conçoit cette mission essentielle du frère enseignant, puis je verrai les principales contributions des Frères des Écoles chrétiennes au mouvement catéchistique. Compte tenu de l'état actuel de mes recherches, moins avancé que prévu, je dois limiter mon exposé au Québec et à la période de 1837 à 1946.

\section{Les «apôtres du catéchisme» $(P i e X)$}

Une- des principales caractéristiques de l'Institut lasallien est son gouvernement centralisé. «À l'exemple de la Compagnie de Jésus, écrit Georges Rigault, l'historien officiel de la congrégation, les Frères des Écoles chrétiennes sont strictement hiérarchisés sous un commandement suprême. Statut personnel de chacun des religieux, nominations et mutations des professeurs, question d'ordre spirituel ou d'ordre matériel concernant les communautés, programmes pédagogiques, tout relève, en dernière analyse, du Supérieur général, tout, dans les grandes lignes, sinon dans le détail, doit aboutir à la Maison-Mère, pour y devenir objet d'étude et de décision de la part du «Régime».2 Dans ce contexte, non seulement les textes officiels - v.g. les Règles communes et Constitutions des Frères des Écoles chrétiennes, la Règle de gouvernement de l'Institut des Frères des Écoles chrétiennes, la Conduite des Écoles chrétiennes, les Douze vertus d' un bon maître - ont-ils une importance primordiale, mais aussi les directives du Très Honoré Frère Supérieur général, telles qu'on les retrouve dans les Circulaires instructives et administratives; de même les décisions des Chapitres généraux qui se réunissent de façon régulière. Voilà pourquoi avant de parler du travail catéchistique des Lasalliens au Canada, il convient de connaître l'idéal proposé par leurs supérieurs.

\section{a. Les vues du fondateur}

L'éducation de la foi est au cœur du projet pastoral de Jean-Baptiste de La Salle. Il fonde son Institut au moment où se développent, en Italie d'abord, puis en France, des mouvements catéchistique et scolaire très liés. Au XVIe siècle, avant même le concile de Trente, le catéchisme aux enfants et aux jeunes gens prend une importance considérable. De même aussi, les «petites écoles» se multiplient. «On leur assigne un objectif

1 Bernard Plongeron, «Qu'est-ce que produire un catéchisme?», Groupe de recherche sur la production des catéchismes de l'Université Laval, La production des catéchismes en Amérique française (XVII-XX ${ }^{e}$ siècles, pp. 83-96.

2 Georges Rigault, Histoire générale de l'Institut des Frères des Écoles chrétiennes, t. V, Paris, Plon, 1945, p. 425. 
apostolique très net, que peuvent définir ces deux termes d' «instruction» et d' «éducation» chrétienne». Alors, dans la pensée des réformateurs comme aux yeux des évêques, «l'école peut et doit constituer un lieu privilégié de la catéchèse aux enfants». 3

Le saint fondateur inscrit son projet dans cette perspective. Son premier biographe, le chanoine Jean-Baptiste Blain, le rappelle bien: pour assurer «ce qui étoit le plus propre pour la propagation de la doctrine Chrétienne \& de la science du salut [...] il s'est proposé deux choses à exécuter. La première d'unir l'éducation à l'instruction dans les Écoles; la deuxième de les peupler $\&$ de les rendre florissantes». ${ }^{4}$ Il se consacre donc totalement à l'œuvre des écoles: il les multiplie et leur donne tous les gages de succès; il leur fixe un objectif global qui fait dépasser la simple instruction religieuse et profane pour tâcher d'éduquer, c'est-à-dire, selon ses propres mots, «de former un Chrétien, \& de lui donner les moïens de mener une vie, qui soit digne de son état, \& du nom qu'il porte».5 Cet objectif est repris dans la bulle In Apostolica dignitatis de Benoît XIII, du 26 janvier 1725: «instruire les enfants [...] de ce qui concerne la manière de vivre honnêtement et chrétiennement».6 Il avait clairement été énoncé dans la première version, restée manuscrite, des Règles communes de l'Institut (1705):

La fin de cet Institut est de donner une education chretienne aux Enfans et cest pour ce sujet qu'on y tient les Écoles afin que les Enfans y etant sous la conduite des maitres depuis le matin jusqu'au soir, ces maitres puissent leur apprendre abien vivre en les instruisant des miseres de notre Religion en leur inspirant les maximes chretiennes et ainsi leur donner l'education qui leur convient. ${ }^{7}$

Pour diriger ces «écoles chrétiennes», Jean-Baptiste de La Salle fonde une communauté de «Maîtres pieux \& habiles»8: habiles sans doute à transmettre le savoir profane et à inculquer les règles du savoir-vivre et de la vie en société 9 , mais plus habiles encore à faire l'éducation de la foi

3 F. Michel Sauvage, Catéchèse et laïcat, Paris, Ligel, 1962, p. 526.

4 Jean-Baptiste Blain, La vie de Monsieur Jean-Baptiste de La Salle, Instituteur des Frères des Écoles chrétiennes, Rome, Maison Saint Jean-Baptiste de La Salle, 1962, p. 358 (Cahiers lasalliens, 8). Reproduction photomécanique de l'édition originale de 1733.

5 Jean-Baptiste de La Salle, Les devoirs d' un chrétien envers Dieu et les moyens de pouvoir bien s'en acquitter, Rome, Maison Saint Jean-Baptiste de La Salle, 1964, préface (Cahiers lasalliens, 20). Reproduction anastatique de l'édition de 1703.

6 «Bulle de N. S.-P. le pape Benoît XIII approuvant les règles et l'Institut des Frères des Écoles chrétiennes», Règles communes et Constitutions des Frères des Écoles chrétiennes, Rome, Maison généralice, 1947, p. VIII.

7 Jean-Baptiste de La Salle, Règles communes des Frères des Écoles chrétiennes, 1705, Rome, Maison Saint Jean-Baptiste de La Salle, 1965, p. 16 (Cahiers lasalliens, 25).

8 Blain, op. cit., p. 359.

9 Jean-Baptiste de La Salle rédige Les Règles de la Bienséance et de la Civilité chrétienne, Rome, Maison Saint Jean-Baptiste de La Salle, 1964, 260p. (Cahiers lasalliens, 19). Reproduction anastatique de l'édition de 1703. 
de leurs élèves. Car, à ses yeux, et compte tenu du contexte de son époque, les frères sont d'abord et avant tout chargés de l'enseignement religieux aux enfants qui viennent à l'école; c'est, répète-t-il à satiété, leur «état», leur «profession», leur «tâche principale», ce à quoi Dieu les destine:

Vous êtes obligés, par votre état, d'apprendre aux enfants les mystères de la religion.

Votre profession vous engage d'apprendre aux enfants la science du salut.

Vous avez dû leur apprendre leur religion; vous y êtes-vous assez appliqués pendant cette année? Avez-vous regardé cette fonction comme votre principal devoir à leur égard?

Vous faites connaître aux enfants les vérités de l'Évangile, ayant été choisis de Dieu pour les leur annoncer. ${ }^{10}$

Le fondateur multiplie les dispositions pour que ses frères remplissent le mieux possible cette mission que l'Église leur confie. L'horaire journalier, par exemple - et plus encore celui du dimanche - prévoit des périodes d'étude du catéchisme. La Conduite des écoles chrétiennes leur prescrit de faire aux enfants une demi-heure de catéchisme par jour, sans compter les séances plus longues («une heure et demie») des dimanches et fêtes; elle détermine aussi la méthode à suivre. Jean-Baptiste de La Salle compose lui-même un Catéchisme des Frères des Écoles chrétiennes qu'il publie en deux parties: Les Devoirs d'un Chrétien envers Dieu (par demandes et réponses) et Les Devoirs d'un Chrétien envers Dieu et les moyens de s'en bien acquiter (par discours suivi). ${ }^{11} \mathrm{Ce}$ dernier texte connaît un succès extraordinaire comme explication du catéchisme par questions et réponses et comme manuel de théologie pour les laïcs. ${ }^{12}$ Enfin, dans ses exhortations et ses écrits, le fondateur ne cesse de rappeler à ses frères les particularités et les obligations de cet aspect de leur vocation. ${ }^{13}$

\section{b. Évolution et continuité}

Après la mort de Jean-Baptiste de La Salle en 1719, l'Institut continue à mettre l'éducation de la foi au cœur de son action, tout en adaptant son projet et ses objectifs aux nouvelles circonstances. Je n'en veux pour

10 Textes cités dans Sauvage, op. cit., pp. 592-600.

11 Jean-Baptiste de La Salle, Les Devoirs d'un Chrétien envers Dieu, T. I et II, Rome, Maison Saint Jean-Baptiste de La Salle, 1964, 2 vol. (Cahiers lasalliens, 20 et 21). Reproduction anastatique de l'édition de 1703.

12 F. Jean Pungier, Jean-Baptiste de La Salle: le message de son catéchisme, Rome, Maison généralice, $1984,214 \mathrm{p}$.

13 Voir tout particulièrement: Jean-Baptiste de La Salle, Méditations pour les Dimanches et les principales Fêtes de l'année, Rome, Maison Jean-Baptiste de La Salle, 1962, 2 vol. (Cahiers lasalliens, 12); Méditations pour le temps de la Retraite, Rome, Maison Saint Jean-Baptiste de La Salle, 1963, IV, 84, 10p. (Cahiers lasalliens, 13). Reproductions anastatiques des éditions originales. 
preuve que la place qu'y tient l'enseignement de la doctrine chrétienne dans les Chapitres généraux et les débats qui s'y tiennent.

Le Chapitre général se réunit, théoriquement, tous les dix ans, mais divers événements peuvent soit en retarder la convocation (les deux guerres mondiales, par exemple), soit l'accélérer (la mort du Supérieur général). ${ }^{14}$ Non seulement les décisions capitulaires, mais plus encore les notes et mémoires, envoyés par les frères profès, et les rapports des commissions nous donnent de précieux indices sur l'état de l'enseignement catéchistique, les objectifs que poursuivent les Lasalliens et les moyens qu'ils se donnent pour atteindre leurs buts. Les Circulaires instructives et administratives des Supérieurs généraux répercutent et expliquent les décisions et, à l'occasion, donnent une nouvelle synthèse de l'action catéchistique des frères des Écoles chrétiennes.

Voici, en gros, ce qui se dégage de la lecture de cette documentation abondante.

«Instruire les enfants de la doctrine de Jésus-Christ, les exercer aux pratiques et aux vertus de la vie chrétienne» demeure toujours le but premier, la fin de l'Institut, un de ses caractères propres, ce que viennent confirmer diverses consécrations romaines dont celle de Pie $\mathrm{X}$ qui décerne aux frères, en 1903, le «titre glorieux» d' "Apôtres du catéchisme».15 C'est que, souligne plus tard le frère Junien-Victor, «peu de congrégations se consacrent aussi formellement à cette mission et se préoccupent, au même degré, de la formation catéchistique de leurs membres».16

Pour mener à bien cette «œuvre capitale»17, l'Institut propose à ses membres des réflexions pédagogiques qui doivent nourrir leur enseignement catéchistique et leur servir de modèle. Pendant longtemps, la Conduite des Écoles chrétiennes demeure le guide incontestable, qu'il faut cependant mettre d'accord avec les progrès de la pédagogie moderne et de la méthodologie. L'édition de 1916 en fait foi dans son chapitre, intitulé «Catéchisme», qui comprend huit parties sur l'importance du catéchisme, la préparation de la leçon de catéchisme, le catéchisme aux différents cours, le temps consacré à cette spécialité dans les écoles chrétiennes (i.e. dirigées par les Lasalliens), la division des prières et du catéchisme, l'étude du saint Évangile, enfin l'obligation et la manière de faire aimer la religion par le catéchisme.

14 Le Chapitre général de 1946 est le $37 \mathrm{e}$.

15 F. Gabriel-Marie, «Pèlerinage à Rome», Circulaires instructives et administratives, 125 (18 oct. 1903), pp. 6-11.

16 F. Junien-Victor, «L'action catéchistique du Frère des Écoles chrétiennes», ibid., 300 (19 fév. 1938), p. 3.

17 Ibid., p. 25. 
Des publications plus spécialisées viennent appuyer ce guide. Au début du $\mathrm{XX}^{\mathrm{e}}$ siècle, les frères Paul-Joseph et Bernard-Louis condensent leur expérience professionnelle dans deux ouvrages qui seront longtemps le vade-mecum des frères enseignants lasalliens. Du premier, les Éléments de pédagogie à l'usage des Frères des Écoles chrétiennes comprennent une partie générale (tome I) sur l'éducation et l'enseignement en général ${ }^{18}$ et une seconde (tome II) consacrée à la méthodologie spéciale où l'auteur consacre 128 pages à l'enseignement de la religion ${ }^{19}$. Après quelques considérations générales, il expose les règles méthodologiques relatives à l'enseignement catéchistique et il en montre l'application par des exemples variés: explications de textes, catéchisme sur un mystère, catéchisme par l'explication de gravures murales, méthode historique, explications de prières liturgiques. Ces pages ont d'abord été publiées dans les Circulaires instructives et administratives de 1901.20

En 1907, le frère Bernard-Louis publie le Manuel du Catéchiste qui, aux yeux de plusieurs, marque «une étape dans l'histoire de l'enseignement du catéchisme».21 L'ouvrage s'inspire à la fois de la méthode de Saint-Sulpice, transmise par saint Jean-Baptiste de La Salle, et de celle dite de Munich, et constitue, pour Mary Coke, avec les premiers livres de l'abbé Charles Quinet, «une sorte de tremplin entre l'enseignement religieux de naguère et la pédagogie nouvelle».22 En 1937, Charles-Eugène Roy le considère encore comme le meilleur guide existant. À noter que le Manuel du Catéchiste, dont on publie un abrégé en 1909, fait partie d'une série de 17 volumes qui constituent le Cours d'instruction religieuse de l'Institut des Frères des Écoles chrétiennes. ${ }^{23}$

Ce Cours sert de base aux études catéchistiques que l'Institut impose à ses membres: «L'étude ordinaire se fera dans les livres de catéchisme publiés par l'Institut», rappelle la Règle de gouvernement. ${ }^{24}$ Ainsi, tous les frères, sauf les directeurs, ceux qui ont 12 ans de profession perpétuelle et ceux qui ont obtenu le diplôme de catéchiste, sont tenus à l'«étude et la récitation journalière du catéchisme», dont on souligne avec vigueur l'obligation aux Chapitres de 1873 et de 1894.25 Des examens, trimestriels

18 Paris, Procure générale, 1901, XX, 442p.

19 Paris, Procure générale, 1902, 514p.

20 «Méthodologie de l'Enseignement de la Religion», Circulaires instructives et administratives, 107 (22 sept. 1901), 132p.

21 Jugement du Centre catéchétique de Louvain, cité dans Junien-Victor, op. cit., p. 25.

22 Mary Coke, Le mouvement catéchétique de Jules Ferry à Vatican II, Paris, Centurion, 1988, pp. 44-45.

23 Voir liste complète dans Junien-Victor, op. cit., pp. 49-50.

24 Règle de gouvernement de l'Institut des Frères des Écoles chrétiennes, Lembecq-lezHal, Maison-Mère, 1923, p. 156.

25 Ibid., p. 157. 
ou à l'occasion de la retraite annuelle, permettent de vérifier les connaissances et même de «dispenser de la récitation quotidienne du catéchisme les Profès dont l'examen aura été très satisfaisant».26

Allant plus loin, le Chapitre général de 1894 institue des examens annuels qui conduisent à un diplôme. Après quelques tâtonnements et expériences, le règlement précise que

1) les profès et les scolastiques (à partir de 1913) peuvent se présenter à l'examien añinuel (29 où 30 déccmbre, depuis 1926);

2) l'épreuve comprend un examen écrit et, jusqu'en 1928, un examen oral;

3) la correction des copies se fait à la maison mère. «Chaque copie, gardant toujours son caractère anonyme, est examinée par deux correcteurs différents et qualifiée selon un barème établi par le Secrétariat. L'annotation des correcteurs est revue et vérifiée par un Bureau, qui détermine les notes définitives».

4) le succès dans cinq cxamens conduit à un Diplôme de catéchiste, Degré Moyen; d'autres examens permettent d'obtenir le Diplôme de catéchiste, Degré Supérieur, avec, selon le cas, mention en apologétique, histoire, Écriture sainte ou ascétisme. 27

Le projet ne connaît pas tout le succès escompté. Après un départ rapide de 1896 à 1900, le nombre des admis diminue considérablement, à cause notamment des bouleversements de 1904 en France et de la guerre 14-18, et, après le conflit, il n'augmente qu'au rythme de la progression du nombre des profès; «dans plusieurs Districts, reconnaît le Supérieur général en 1938, nos jeunes sujets semblent se désintéresser trop facilement des examens de catéchisme, une fois qu'ils se trouvent placés en communauté». 28

Les autorités lasalliennes tiennent également à ce que les frères appliquent dans l'enseignement religieux les meilleures méthodes pédagogiques. Les directives générales sont explicitées, de façon générale, dans les Circulaires instructives et administratives; périodiquement, un numéro plus élaboré fait la synthèse des directives et donne un élan à de nouvelles initiatives. Tels sont:

- F. Gabriel-Marie, «La méthodologie de l'enseignement de la Religion», 107 (22 sept. 1901), 132p.

26 Chapitres généraux de l'Institut des Frères des Écoles chrétiennes, Paris, MaisonMère, 1902, p. 149. Il s'agit d'une décision du Chapitre général de 1882.

27 Ibid., pp. 55-57.

28 Ibid., p. 133. 
- F. Junien-Victor, «L'Action catéchistique du Frère des Écoles chrétiennes», 300 (19 fév. 1938), 178p.

- F. Athanase-Émile, «Résultats du Chapitre général», 318 (16 juillet 1946), pp. 82-86

- F. Nicet-Joseph, «La mission de Catéchiste du Frère des Écoles Chrétiennes», 371 (2 fév. 1962), 318p.

Plus stimulants encore sont les commentaires critiques que les profès font dans leurs notes et mémoires soumis aux Chapitres généraux. Même s'ils décrivent ordinairement - et parfois de façon assez brutale - une situation restreinte (celle de leur district, le plus souvent), ils amorcent des discussions fécondes et permettent aux commissions d'apporter des nuances et de faire des suggestions qui aboutissent à la politique dont nous venons de parler.

En 1873, par exemple, le frère Irlide, qui sera élu supérieur général plus tard, analyse ainsi la situation:

À propos des études que l'on doit faire dans toutes nos maisons, il convient de signaler l'étude du Catéchisme: on perd le temps, on travaille sans suite et sans intelligence, et les catéchismes que l'on fait dans les Communautés le jeudi et le dimanche ne profitent ni à la science, ni à la méthode.

On est étonné, et péniblement impressionné, quand on voit à quel point un très-grand nombre de Frères sont ignorants de la science de la Religion.

À part quelques définitions, la plupart des Frères sont incapables de rien dire sur la branche la plus importante de l'enseignement. Dès qu'on les sort de la lettre, ils restent muets ou disent des choses peu sensées. 29

En 1894, un frère d'Italie note lui aussi des lacunes: "Que l'instruction religieuse des Frères ne soit pas, en général, tout-à-fait à la hauteur de leur mission, surtout en ces temps-ci, c'est une chose assez reconnue et déplorée par nos vigilants supérieurs»; il met surtout le doigt sur les aspects négatifs de la demi-heure réglementaire d'étude du catéchisme:

Je suis d'avis que la demi-heure réglementaire d'étude du catéchisme, considérée comme un exercice de piété, à l'instar de la lecture spirituelle et de la méditation, est plus nuisible qu'utile, et en voilà les principales raisons:

$1^{\circ}$ Tout ce qui est commandé ou défendu soulève toujours la rébellion du viel-homme: ceci tient à la nature même de l'homme. [...]

$2^{\circ}$ [Une] Demi-heure par jour ne suffit pas à de jeunes frères qui ont besoin d'apprendre au plus vite beaucoup de choses pour se mettre à même de bientôt faire le Catéchisme comme il faut; et pourtant à cause de la raison dite en premier lieu nous sommes tous portés à retrancher plutôt qu'à ajouter au temps des exercices de piété.

29 F. Irlide, Notes pour le Chapitre général, 1873, Archives des Frères des Écoles chrétiennes à Rome [AFECR], ED 230, 3. 
$3^{\circ}$ Il arrive assez souvent des journées dans lesquelles un jeune frère est tellement accablé par des pensées de classe ou d'études profanes, que les plus belles résolutions viennent échouer contre le miroitement de l'imagination exaltée. Tout ce que le pauvre frère peut faire alors pendant l'étude du catéchisme c'est de promener son livre en combattant les distractions autant que possible; et après cela il ne se croira nullement obligé de prendre un autre temps pour refaire son étude de catéchisme. ${ }^{30}$

Au même Chapitre, quelques autres mémoires se plaignent «de l'insuffisance de certains maîtres, même un peu anciens», mais la Commission sur la formation intellectuelle et religieuse «trouve fausse l'appréciation de ces notes comme étant trop générale» et «ne croit pas l'Institut trop audessous de sa tâche». Ce qui ne l'empêche pas de proposer et de faire voter l'inauguration des examens qui doivent conduire aux diplômes de catéchiste. ${ }^{31}$

Plusieurs des 45 notes soumises au Chapitre de 1934 à propos des études religieuses confirment «une désaffection, vis-à-vis des Examens de Catéchisme, de la part de nombreux Frères vivant en Communauté»; un texte bicn documenté avance quatre facteurs d'explication: l'insuffisance des moyens intellectuels, une décision trop tardive pour la préparation immédiate de l'examen, le peu de temps consacré aux études religieuses et la perte de temps. La Commission chargée d'étudier ces mémoires propose un lot de réformes, que les capitulants entérinent. ${ }^{32}$

Enfin, en 1946, si quelques notes (surtout de l'Amérique latine et de l'Espagne) parlent encore d'une certaine désaffection pour le catéchisme au profit des études profanes spécialisées, la Commission ad hoc rend «un juste hommage» au programme élaboré par le Chapitre de 1934 et conclut que «l'élan donné a été suivi par de nombreux Frères». Son rapport traite largement de la formation catéchistique des frères et de l'organisation des études religieuses dans les classes. Le tout est relié de près à un nouvel essor de l'enseignement de la pédagogie et à un renouveau général des études. 33

En présentant, en 1947, une rétrospective du "Cinquantenaire de nos examens d'instruction religieuse (1896-1946)», le Supérieur général Athanase-Émile insiste surtout sur «le travail, la constance et les efforts que cela représente»:

30 F. Ubald, Instruction religieuse, 7 oct. 1894, p. 8-9, ibid., ED 232, 6.

${ }^{31}$ Rapport de la Commission de Formation Intellectuelle et Pédagogique, 1894, pp. 34, ibid., ED 232, 12.

32 Rapport de la troisième Commission du Chapitre général, 1934, 9p., ibid., ED 237, 3; F. Junien-Victor, «Résultats du Chapitre général», Circulaires instructives et administratives, 283 (6 août 1934), pp. 29-37; F. Junien-Victor, «Examens de catéchisme. Nouveaux programmes du Cours supérieur», ibid., 288 (19 mars 1935), $71 \mathrm{p}$.

33 F. Athanase-Émile, «Résultats du Chapitre général», ibid., 318 (16 juillet 1946), pp. 82-86. 
De nombreux jeunes frères ont trouvé dans ces études les lumières qui les ont soutenus dans les moments difficiles; d'autres, d'âge mûr, se sont enthousiasmés pour leur belle mission et, en fortifiant leurs convictions, sont devenus de vrais Apôtres du Catéchisme; beaucoup de nos Frères Directeurs ont puisé dans nos volumes les idées-forces qui ont corsé leurs entretiens du Dimanche.

De plus, à son avis, «dans le domaine de la composition d'ouvrages de vulgarisation, de cours de méthodologie, de conférences ou d'exposition catéchistique, notre Institut a pris dans ces derniers temps une place de premier plan». ${ }^{34} \mathrm{Ce}$ verdict s'applique assez bien, je crois, à la situation dans les districts du Canada.

\section{La contribution des Frères des Écoles chrétiennes au mouvement catéchistique au Canada}

Appelés à Montréal par les Sulpiciens et Mgr Jean-Jacques Lartigue, les Lasalliens y développent ce que l'évêque appelle «des Écoles vraiment Chrétiennes», c'est-à-dire celles «où l'on ne se contente pas de donner l'instruction littéraire», mais où «on y insiste principalement sur ce qui forme la jeunesse aux bonnes mœurs et à la piété». 35 Ils ont pleines facilités d'agir à leur guise et d'imposer leurs programmes, leurs horaires, leurs méthodes et leurs manuels, puisque, dans de nombreux cas, surtout au début, ils dirigent des écoles privées et que, même après la création du réseau public, il faut attendre plusieurs décennies avant que le Conseil de l'Instruction publique et les Commissions scolaires exercent un contrôle efficace sur l'enseignement primaire. Règle générale, les frères ont donc toute liberté de vivre leur vocation et d'implanter leur modèle d'éducation.

\section{a. Les conséquences générales de leur arrivée}

Au point de vue de l'éducation de la foi et de l'enseignement catéchistique, comme au point de vue de l'enseignement en général, l'arrivée des Frères des Écoles chrétiennes marque une ère nouvelle.

Dans un Québec français où à peu près tout était à construire au point de vue scolaire - seule la Congrégation de Notre-Dame possède alors une certaine tradition pour l'éducation des filles -, l'enseignement des frères va longtemps servir de modèle au primaire. Dans les couvents comme dans beaucoup d'écoles dirigées par des laïques, on retrouve cette atmosphère religieuse, cet horaire ponctué de prières, cette discipline rigide, même cette méthode simultanée d'enseignement qui caractérisent la pédagogie lasallienne. C'est que, dès leur arrivée, les frères ont

34 F. Athanase-Émile, «Cinquantenaire de nos Examens d'instruction Religieuse (18961946)», ibid., 323 (21 nov. 1947), pp. 8-11.

35 Mgr Lartigue, «Mandement», 12 mars 1839, MEM, 1, pp. 48-49. 
libéralement distribué des exemplaires de la Conduite des Écoles chrétiennes. De plus, sans ouvrir une école normale, comme l'a souhaité un temps $\mathrm{M}^{\mathrm{gr}}$ Lartigue, ils ont donné des cours à des religieuses - les Sœurs des Saints Noms de Jésus et de Marie, par exemple - et à des laïques regroupés à Montréal, à Québec et à Trois-Rivières. Ce qui permet au frère Réticius, plus tard, de lancer cette pointe au principal de l'École normale Jacques-Cartier de Montréal, l'abbé Anthelme-Hospice Verreau: «Et ces cours normaux, pour être moins éclatants que ceux qui les ont remplacés, n'en étaient pas moins utiles». Plus modestes, plus économiques et plus religieux, mais néanmoins solides et pratiques, comme l'a souligné le surintendant Jean-Baptiste Meilleur. Il faut aussi ajouter les nombreux sujets qui, après avoir quitté la communauté, «prennent généralement place parmi les maîtres laïques où il se font bientôt une honorable position et disposent en faveur de l'éducation des connaissances et de l'expérience qu'ils ont acquise [sic] dans l'état religieux».36

Enfin, en certains endroits, des prêtres ont tenu à imiter l'enseignement particulièrement brillant de quelques frères; ce geste d'humilité (!), rapporté dans des Historiques de communautés, n'est pas répandu dans une Église enseignante qui tient garder à leur place des gens qui ne savent même pas le latin...

Les Frères des Écoles chrétiennes apportent aussi une contribution notable en publiant des manuels classiques. Dès 1841 est mis en vente le Nouveau traité des devoirs du chrétien envers Dieu qui connaît un immense succès. Notre groupe de recherche en a recensé 28 éditions différentes au Québec. Vanté de toutes parts pour ses qualités pédagogiques et humaines - «[...] les premiers chapitres du Devoir du chrétien, dans la collection des Frères, sont de véritables chefs-d'œuvre d'analyse, écrits avec une touchante simplicité», constate P.-J.-O. Chauveau en 1855 - et destiné aux élèves les plus avancés, il est, jusqu'aux années 1880 , le manuel de lecture le plus répandu; en 1855, par exemple, le surintendant de l'Instruction publique le retrouve dans 193 municipalités. Le tout aussi célèbre Syllabaire des frères constitue lui aussi un «précis de la doctrine chrétienne».37

À partir de 1869, les frères mettent sur le marché un certain nombre de catéchismes expliqués. La Série de questions sur les principales vérités de la religion (24p.) est un outil pédagogique destiné aux maîtres pour leur permettre de mieux appliquer une règle fondamentale de la Conduite des

36 F. Réticius, Aux Honorables Membres du Comité catholique du Conseil de l'Instruction publique, 14 sept. 1884, pp. 15-16, 22.

37 P.-J.-O. Chauveau, Rapport sur l'instruction publique dans le Bas-Canada pour l'année 1855 , p. 14. 
écoles: «L'enseignement par demandes et par réponses s'emploie pour toutes les spécialités, mais principalement pour le catéchisme» et «les sous-questions doivent avoir pour objet le sens des mots, avant de porter sur celui des propositions».38 Vers 1878 paraît le Manuel du catéchiste ou Questionnaires avec réponses sur la doctrine chrétienne (179p.) et, en 1879, le Questionnaire du petit catéchisme de la province ecclésiastique de Québec (370p.). Attribués au frère Adelbertus, l'un des quatre pioniers de 1837, ces manuels s'adressent aux catéchistes, «à ceux surtout qui débutent dans la carrière de l'enseignement» et leur proposent des séries de questions, des pratiques et des histoires. Les Lasalliens se font aussi les propagandistes du catéchisme expliqué d'un de leurs amis, le sulpicien Amans-Théophile-Marie Luche, qui publie en 1897 les Notes d'un catéchiste ou Court commentaire littéral sur le Catéchisme des provinces ecclésiastiques de Québec, Montréal, Ottawa (708p.). Ces manuels connaissent un certain succès et sont réédités quelques fois, mais ils cèdent la place à deux best sellers: Le Code catholique ou Commentaire du Catéchisme des provinces ecclésiastiques de Québec, Montréal et Ottawa de l'abbé David Gosselin et l'Explication littérale et sommaire du catéchisme de Québec, Montréal et Ottawa d'Édouard Lasfargues, des Frères de Saint-Vincent de Paul. Néanmoins, en 1929, le frère Macarius-Léo donne une nouvelle version du Questionnaire sur les principales vérités de la religion: d'après le texte du catéchisme des provinces ecclésiastiques de Québec, Montréal et Ottawa (101p.). Enfin, il ne faut pas l'oublier, de nombreux frères rédigent pour leur propre gouverne de véritables catéchismes expliqués ou des recueils d'histoires ou d'exemples, qui sont demeurés manuscrits quand ils n'ont pas tout simplement disparu.

Au fil des décennies, cependant, l'effervescence des origines cède la place à la quiétude institutionnelle. Le frère Réticius sonne l'alarme en 1880 , en comparant les communautés canadiennes aux meilleures de France; le tableau est sombre:

[...] nous avons peu de sujets cultivés; quelques individualités percent dans la sphère des mathématiques; pour les autres spécialités, nous demeurons dans la médiocrité et au-dessous; le niveau de la culture intellectuelle est inférieur à celui de nos frères de France. Ce n'est pas manque d'intelligence chez les Canadiens; non, c'est plutôt défaut de culture, de travail méthodique. [...]

[...] nos classes, quoique les meilleures, sont néanmoins fort médiocres. On y trouve généralement un ordre et une discipline convenables, mais qui sont plutôt un effet du caractère des petits Canadiens, que de l'habileté des maîtres [...] Interrogez un enfant sur son devoir, sa lecture, son catéchisme; demandez-lui le sens de tel mot, le pourquoi de telle chose, son silence ou sa balourdise vous montre la valeur professorale du maître.

38 Conduite à l'usage des Écoles chrétiennes, Versailles, Beau, 1870, pp. 11-12. 
Même les manuels de la communauté, «les meilleurs quand ils étaient les seuls», sont, dit-il, «au-dessous de ceux en usage dans les écoles laïques». Quant à la science pédagogique, elle est «nulle» ${ }^{39}$, juge-t-il péremptoirement.

Il y a sans doute une certaine dose d'exagération dans ces propos apôtre d'une meilleure formation intellectuelle et religieuse des frères, le frère Réticius a naturellement tendance à critiquer ses subordonnés - , mais il y a aussi beaucoup de vrai. Les frères eux-mêmes admettent qu'ils ont parfois vu trop grand ou trop facilement cédé aux demandes de fondation et que la qualité n'a pas toujours suivi la quantité. Ce mal est dénoncé pour l'ensemble de l'Institut au Chapitre général de 1873, qui impose un moratoire sur les nouvelles fondations et propose même la fermeture de certaines maisons. C'est dans ce sens qu'agissent, en Amérique, les frères visiteurs Armin-Victor et Réticius, mais leur action ferme ne corrige pas toutes les faiblesses et le frère Robert peut encore écrire en 1940: «Ici même, au Canada, n'a-t-on pas cédé parfois au zèle imprudent qui nous porte à fonder des œuvres trop vastes et à multiplier les ouvriers sans les avoir préparés suffisamment. On a dû improviser des chefs et on a confié à des maitres riches de leur seule bonne volonté, des tâches audessus de leurs forces. Or, même aux yeux du Seigneur, la bonne volonté ne suffit pas pour que les œuvres prospèrent $[\ldots ..] \gg .40$

Les frères sont cependant poussés par leurs supérieurs du Canada, et nommément le frère Réticius lui-même, de 1880 à 1913, à titre de visiteur puis d'assistant, et stimulés par divers événements comme l'arrivée des frères français en 1904-1907, le développement général des études, la possibilité de s'inscrire au Diplôme d'enseignement moderne et de pédagogie (lancé à l'Université de Montréal en 1922) et la fondation de l'Institut pédagogique Saint-Georges en 1929. Tout cela crée un climat d'ouverture qui permet de mieux recevoir les directives des supérieurs et Chapitres généraux, d'en essayer la mise en application et de se perfectionner en science religieuse en se présentant aux examens de catéchisme de l'Institut. Ceux-ci connaissent une grande popularité au Canada qui, jusqu'en 1914, est de loin le district qui présente le plus grand nombre de candidats et obtient le plus de diplômes. Même s'il n'y a pas de déclin véritable dans les années 1920 et au début des années 1930, on note une progression exceptionnelle dans le nombre des inscrits après les célébrations du centenaire de 1937, quand les autorités proposent comme un des

39 F. Réticius au F. Irlide, 30 oct. 1880, AFECR, NO 111, 2.

40 F. Robert, «Le Rayonnement du Frère des Écoles chrétiennes par sa Compétence Générale», Les études, V, déc. 1940, p. 73. Cette publication est l'organe officiel du Bureau des études du district de Montréal. 
fruits spirituels de ces célébrations l'essor des études religieuses et profanes. La «légion du centenaire» concerne davantage le Diplôme d'enseignement moderne et de pédagogie, mais a un effet d'entraînement pour les examens de catéchisme. Dans le district de Montréal, par exemple, le nombre des inscrits monte en flèche jusqu'en 1941, puis baisse lentement.

\section{District de Montréal Examens de catéchisme de l'Institut, 1936-1946}

$\begin{array}{lrrrr}\text { Année } & \text { Inscrits } & \text { Présentés } & \text { Échecs } & \text { Diplômes } \\ 1936 & 62 & 41 & 10 & 3 \\ 1937 & 73 & 47 & 16 & 2 \\ 1938 & 132 & 74 & 17 & 2 \\ 1939 & 163 & 117 & 19 & 2 \\ 1940 & 222 & 145 & 16 & 1 \\ 1941 & 236 & 158 & 25 & 7 \\ 1942 & 211 & 151 & 14 & 5 \\ 1943 & 203 & 163 & 25 & 15 \\ 1944 & 186 & 132 & 12 & 11 \\ 1945 & 186 & 104 & 21 & 3 \\ 1946 & 171 & 108 & 23 & 5\end{array}$

SOURCE: Examens de Catéchisme. Y a-t-il un réel danger d'inflation!, s.d. (1949), Archives des Frères des Écoles chrétiennes à Québec, 8B, Foyer catéchétique, E-3.

On peut observer le même phénomène à Québec et à Toronto. Les supérieurs sont les premiers à se plaindre qu'un tiers des inscrits ne se présentent pas aux examens. De plus, comme les frères doivent se présenter plusieurs années de suite aux examens, plusieurs abandonnent en cours de route, d'où le petit nombre de diplômés. Le nombre des échecs, quant à lui, se situe à peu près dans la moyenne de l'ensemble de l'Institut (18,6\% comparé à $15 \%$ environ). Cette popularité nouvelle des examens de catéchisme est d'autant plus notable qu'elle coöncide avec une participation importante des Frères des Écoles chrétiennes au mouvement méthodologique qui secoue alors le monde de la catéchèse au Québec.

\section{b. La participation des Frères des Écoles chrétiennes au mouvement méthodologique}

Ce mouvement de réforme prend son essor dans les années 1930, même si on n'a pas attendu jusque-là pour critiquer le catéchisme officiel et la sclérose de l'enseignement catéchistique. Dès le début du $\mathrm{XX}^{\mathrm{e}}$ siècle, 
plusieurs membres du clergé, dont certains évêques, proposent le remplacement du catéchisme de 1888; l'idée reçoit de nouveaux appuis quand Rome fait des pressions pour faire adopter le Catéchisme catholique du cardinal Gasparri comme catéchisme unique pour l'ensemble de l'Église. ${ }^{41}$ Depuis longtemps d'ailleurs, les milieux scolaires, et nommément les Frères des Écoles chrétiennes, dénoncent le Catéchisme des provinces ecclésiastiques de Québec, Montréal, Ottawa comme «ce que l'on peut imaginer de plus abstrait, de plus aride, de loin le manuel le plus terne entre les mains des écoliers».42

L'enseignement religieux lui-même est critiqué comme «trop théologique, livresque et mécanique».43 Particulièrement à partir de 1930 , enseignants et pédagogues le dénoncent et ils trouvent un solide écho de leurs revendications dans un mémoire que M $^{\mathrm{gr}}$ François-Xavier Ross, de Gaspé, présente à ses collègues en 1933. Intitulé Faut-il un nouveau catéchisme? Quelle méthode?, le texte, qui sera publié en brochure, ne cache aucune faiblesse: le "petit catéchisme» est sans doute «un bon et substantiel résumé de la doctrine chrétienne», mais il ne présente pas Jésus comme une personne encore vivante parmi nous; sa méthode «synthétique et déductive» comporte des «formules abstraites, trop techniques, trop théoriques»; surtout, la fâcheuse habitude de le faire mémoriser est plutôt «un délit contre la raison».44 Ses propos sont largement repris dans les années suivantes par l'abbé Charles-Eugène Roy, l’abbé Irénée Lussier, le père Alcantara Dion, sœur Saint-Ladislas qui se font les apôtres d'une méthode plus inductive.

Les Frères des Écoles chrétiennes sont bien placés pour entrer dans ce mouvement de renouveau et promouvoir ce qu'on va appeler l'enseignement vivant du catéchisme. Déjà éveillés au problème par les textes et la réflexion demandée par l'Institut, plusieurs d'entre eux vont s'initier à la pédagogie nouvelle en Belgique et, dès son ouverture en 1929, l'Institut pédagogique Saint-Georges œuvre dans la même veine. Les scolasticats et noviciats sont bien au fait des initiatives européennes (France, Belgique,

41 Jean-Paul Rouleau, «La production du Catéchisme catholique, édition canadienne (1951)», Raymond Brodeur et Jean-Paul Rouleau, Une inconnue de l'histoire de la culture. La production des catéchismes en Amérique française, Québec, Anne Sigier, 1986, 319-320.

42 F. Antonin, Le foyer documentaire catéchétique de Sainte-Foy, p. 2, Archives des Frères des Écoles chrétiennes à Québec [AFECQ], BA, Foyer catéchétique, B-10.

43 Raymond Anctil, «L'enfant prend la parole: un tournant récent de notre histoire catéchétique», Société canadienne d'histoire de l'Église catholique, Sessions d'étude 46 (1979), p. 33.

44 Mgr Ross, Faut-il un nouveau catéchisme \& Quelle méthode?, document annexé au Procès-verbal de la réunion des archevêques et évêques de la province civile de Québec, 1933, Archives de l'archevêque de Rimouski. 
Italie) et se servent de ces exemples pour appuyer un mouvement semblable au Québec. Vers 1936, le frère François, de Sainte-Foy, fonde un Foyer documentaire catéchétique où il rassemble une masse de matériel didactique dans un local du noviciat; «avec cinq confrères enthousiastes», il diffuse dans le district les idées, les livres et le matériel. C'est là que naît l'idée des semaines d'enseignement catéchistique.

Elle est une suite de l'appel du frère Supérieur général de 1938. «Le Très Honoré Frère Junien-Victor venait de nous communiquer la circulaire sur l'enseignement religieux» où il se demande «si vraiment le catéchisme donné par ses fils est un enseignement VIVANT, INTÉRESSANT, tel que le préconisait saint Jean-Baptiste de La Salle». Le frère François songe alors à organiser une semaine catéchistique «où seraient mis à contribution tous les moyens susceptibles de donner à l'enseignement de la religion son intérêt maximum».45 Le comité d'organisation choisit d'abord la semaine du 6 au 15 mai 1939 pour la tenue de l'événement, mais, comme elle coïnciderait avec «la visite de nos augustes souverains, le roi et la reine d'Angleterre», elle est reportée en octobre. ${ }^{46} \mathrm{Ce}$ délai permet de donner plus d'ampleur à la semaine pédagogique et de la préparer par une série d'articles publiés dans l'organe de la Commission des Études du District de Québec, La Voix du travail.

La Semaine de l'enseignement religieux vivant s'ouvre à l'Académie de Québec le vendredi 13 octobre 1939 sous la présidence du cardinal Jean-Marie Villeneuve (ancien élève des frères à Montréal) et se poursuit jusqu'au 22 octobre. Elle comporte une exposition catéchistique et la projection de quelques films, des conférences (aucune par les frères) et des séances de catéchismes modèles faites par les frères Georges et Omer of Mary de Montréal. Dans un numéro spécial de La Voix du Travail, le frère visiteur provincial Natalus-Hébert donne la signification du terme «catéchisme vivant» qu'on affiche depuis un an, dit-il, «comme une oriflamme de croisade»:

Plus vivant dans sa forme expositive, qui fait appel aux procédés intéressants, aux méthodes intuitives, aux gravures, aux graphiques, à la lumière de l'écran; plus attrayant pour l'écolier qui n'est plus un auditeur aux bras croisés, mais un acteur dans un chœur parlé...

Plus vivant dans sa fin qui ne se limite pas à l'exposition doctrinale, mais vise à former le chrétien dans sa VIE de tous les jours.

45 F. Romuald-Hosea, «L'enseignement religieux vivant - Le T. C. F. Assistant approuve... loue... stimule», La Voix du travail, VI, 7 (13 déc. 1941), p. 81.

46 F. François, «Pour un enseignement religieux vivant», ibid., III, 11 (20 mars 1939), p. 100. 
Plus vivant, c'est-à-dire catéchisme pour la VIE et non pour l'examen, pour la vie concrète et ses conséquences palpables... ${ }^{47}$

La semaine connaît un grand succès. Les conférences attirent des centaines de personnes - 1200 à celle du père Schelpe au Palais Montcalm - et les journaux les résument largement; des milliers de visiteurs font le tour de l'exposition. Pour prolonger les effets de la rencontre, plusieurs auditeurs proposent et vont voter des vœux dont on peut souligner celui-ci: «IL EST PROPOSÉ qu'une requête délicatement, humblement mais instamment suppliante soit présentée à l'Ordinaire, pour obtenir au moins une présentation plus pédagogique du «CATÉCHISME DIOCÉSAIN».48 Mais la conséquence la plus marquante est sans doute, chez les frères, un élan nouveau pour l'enseignement du catéchisme vivant, dont font régulièrement écho les organes des commissions des études de Québec et de Montréal, La Voix du travail et Les Études.

Le Foyer documentaire catéchétique de Sainte-Foy rayonne un peu partout. À l'été 1940, deux frères sont invités à Rimouski pour donner des cours de méthodologie catéchistique au personnel enseignant; du 19 au 21 juillet, les Sœurs du Saint-Rosaire font une exposition des travaux catéchistiques de 45 de leurs écoles. ${ }^{49}$ Les frères sont aussi demandés à l'Université du Sacré-Cœur de Bathurst pour participer, du 15 au 18 juillet 1941, au Congrès d'enseignement catéchistique et y présenter leur exposition. À Trois-Rivières, du 24 au 31 octobre 1941, ils organisent une semaine d'enseignement religieux pour «faire connaître les différentes méthodes catéchistiques et donner des modèles de ce qu'on peut appeler l'enseignement vivant de la doctrine chrétienne».50

Cependant, c'est dans le district de Montréal que le mouvement prend la plus grande ampleur. On y lance la Croisade du catéchisme qui s'étend de novembre 1940 à novembre 1941 et qui mobilise les directeurs, les frères, les sujets en formation et même les grands élèves. On lui fixe un quadruple but: stimuler l'étude du catéchisme parmi les frères; perfectionner les méthodes d'enseignement du catéchisme; intéresser les élèves à l'étude du catéchisme; former des catéchistes volontaires parmi les grands élèves (c'est-à-dire exercer les grands élèves à l'explication du catéchisme aux plus jeunes). Préparée de longue main par un Comité catéchistique mis sur pied en septembre 1939, qui publie ses procès-verbaux et ses directives dans Les Études, animée par le frère Georges d'une vitalité et d'un dynamisme exceptionnels, la croisade comporte la réalisation de

47 F. Hébert, «Le mot de la fin...», ibid., IV, 3 (2 oct. 1939), p. 31.

48 F. Clovis, «Clôture de la SEMAINE d'enseignement religieux, VOEUX émis», ibid., IV, 5 (1 nov. 1939), p. 53.

49 «Exposition catéchistique à Rimouski», L'Action catholique, 21 juillet 1940.

50 Coupures de journaux, AFECQ, 8A, Foyer catéchistique. C-5. 
deux plans (A: le milieu local; B: le district), la tenue de deux réunions générales, des articles de fond dans l'organe de la Commission des études, des cours de vacances et, comme couronnement, une Semaine d'enseignement religieux qu'on offre comme participation des Frères des Écoles chrétiennes aux festivités du troisième centenaire de Montréal. Elle se tient au Mont-Saint-Louis du 14 au 23 novembre 1941 et, grâce à la collaboration des journaux et de la radio, devient un événement médiatique. Le programme est semblable à celui de Québec, avec cette différence que presque tous les conférenciers sont des frères des Écoles chrétiennes. L'exposition catéchistique présente le matériel du Foyer catéchétique de Québec, la collection du père Simon Chênevert, o.m.i., curé de la paroisse Saint-Pierre de Montréal et les travaux des élèves des Sœurs du Saint-Rosaire de Rimouski. Simultanément, du 19 au 22 novembre 1941, se tiennent à l'Académie De-La-Salle d'Ottawa des Journées de pédagogie catéchistique qui reçoivent quelques-uns des conférenciers de Montréal et, plus tard, l'exposition. ${ }^{51}$

Selon tous les témoignages, l'événement connaît un «succès extraordinaire ${ }^{52}$ et, pour en perpétuer les effets et le souvenir, on publie les textes des conférences et des ateliers de travaux pratiques, agrémentés de plusieurs illustrations. ${ }^{53}$ Pour que les efforts d'amélioration de l'enseignement catéchistique se continuent, les frères organisent chaque été des cours de méthodologie; dans la même veine, les écoles sont invitées à préparer une exposition annuelle dont on regroupe les meilleurs matériaux à une exposition catéchistique tenue au Mont-de-La-Salle. ${ }^{54}$ Plusieurs frères sont aussi mobilisés pour la production de matériel didactique. Les frères Merry-Alphonse, Major-Alexandre et Raymond-Joseph publient huit séries de Catéchismes volants qui servent à la fois d'ouvrage de préparation pour les maîtres et de manuel pour les élèves; les fiches volantes, disent leurs auteurs, «obvient à la routine qui accompagne un manuel longtemps usagé» et «simplifient le texte d'un manuel nécessairement abstrait dans son exposé».55 Plus directement reliée à la semaine catéchistique de 1941, la production des cahiers d'exercices (avec guides du maître), intitulés Mon cahier de religion, fait appel à une armée de frères des diverses maisons du district de Montréal. Après plusieurs mois de tâtonnements, le frère Cyrille, qui a fait ses preuves avec l'Oeuvre d'un

51 Programme général - Semaine d'Enseignement Religieux, AFECM, T7C4lc.

52 F. Georges au F. Nivard-Anselme, 12 déc. 1941, ibid., T7C41b.

53 L'Apostolast catéchistique - Notes pédagogiques sur l'enseignement religieux Souvenir de la Semaine d'enseignement religieux de Montréal, Montréal, Les Frères des Écoles chrétiennes, 1942, 198p.

54 F. Cyrille, «Reportage sur l'Exposition catéchistique du Mont-de-La-Salle», Les Études, VII, 1 (sept. 1942), pp. 19-24.

55 Catéchismes volants, $3^{\mathrm{e}}$ série, Montréal, Les Frères des Écoles chrétiennes, 1940, p. 3. 
siècle, prend charge du projet et le mène rapidement à terme: «[...] je commençai, dit-il, la préparation d'une série allant de la $3^{\mathrm{e}}$ à la $9^{\mathrm{e}}$ année. Chaque communauté fut invitée à la préparation d'un chapitre du catéchisme d'alors, suivant les modèles que j'avais préparés pendant que des Frères spécialistes en liturgie, action catholique, histoire sainte, évangile, etc., montaient leur section». Mis sur le marché en 1944, ces cahiers connaissent un succès très rapide. Le frère Cyrille se vante-t-il quand il note dans ses mémoires? «Ces cahiers nouveau genre et très pratiques furent utilisés à près de deux mıllions d'exempiaires et traduits deux fuis en anglais, deux fois en espagnol et même en tamoul et en cingalais, dans la lointaine Île de Ceylan». 56

Tous ces efforts - et bien d'autres - sont faits, assurent les frères, pour «assurer à notre Congrégation la place qu'elle occupe - la première — dans l'enseignement du catéchisme». 57

\section{Conclusion}

Au Canada comme ailleurs, les Frères des Écoles chrétiennes veulent être des «apôtres du catéchisme», c'est-à-dire les premiers, les meilleurs (!) de ces intermédiaires obligés qui expliquent, font apprendre, interprètent aux enfants les catéchismes abstraits et trop souvent abscons produits par des théologiens plus imbus de science que de pédagogie. Ils arrivent en 1837, riches d'une longue tradition et d'outils pédagogiques qui leur permettent de jouer un rôle primordial dans le développement de l'enseignement du catéchisme.

Graduellement, cependant, la multiplication des maisons, qui font peser un poids exorbitant sur leurs épaules, la préparation trop rapide et insuffisante des candidats à l'enseignement, un climat général scolaire rien moins que progressif font que plusieurs frères négligent leur principale «mission» ou du moins glissent dans la routine et ne sont plus les leaders qu'ils devraient être. Les rappels fréquents et surtout les coups de semonce des autorités centrales peuvent en réveiller quelques-uns, mais il faut attendre les années 1930-1940 pour voir les Lasalliens appuyer de toutes leurs forces le mouvement de renouveau méthodologique dans l'enseignement catéchistique, multiplier les initiatives, se faire les apôtres de l'enseignement religieux vivant et produire de nouveau des outils pédagogiques populaires.

56 F. Meldas-Cyrille, «Mouvement catéchistique. Deux pages d'histoires», ibid., VII, mars 1943, pp. 170-174; Sur ma route. Notes historiques et anecdotiques (1895-1972), p. 65.

57 «La croisade du catéchisme», Les Études, V, fév. 1941, p. 149. 
Mais il ne faudrait pas croire que, pour les frères, l'éducation de la foi se limite à l'enseignement du catéchisme. En plus d'assurer dans leurs classes un climat religieux, ils mobilisent les élèves dans des associations destinées, entre autres buts, à assurer leur persévérance dans la foi: Congrégation de la Sainte-Vierge, Société des Amis du Sacré-Cœur de Jésus, Congrégation des Saints-Anges, Archiconfrérie du Très-Saint-EnfantJésus; plus tard, les Cadets du Sacré-Cœur, la Croisade eucharistique... Les amicales, qui deviennent un moment si populaires, doivent aussi, théoriquement, joưer un rôle semblable. Plusieurs frères s'intéressent aussi à l'Action catholique. D'abord à l'Action catholique générale des années 1910-1920: on connaît l'expérience exceptionnelle du frère MarieVictorin, qui, dans le cercle La Salle, regroupe les grands élèves et les anciens du Collège de Longueuil et nourrit leur foi par l'étude, la réflexion et les retraites fermées. Puis, à la fin des années 1930 et dans les années 1940, l'Action catholique spécialisée (JEC) dont l'expansion coöncide avec la croisade de l'enseignement religieux vivant. Enfin, les frères vont participer de plus en plus à des œuvres extérieures, comme le scoutisme, les colonies de vacances... C'est ainsi qu'ils essaient de répondre à l'appel de leur fondateur de former des chrétiens et de leur donner les moyens de vivre leur foi. 